\title{
Glutathione transferase kappa deficiency causes glomerular nephropathy without overt oxidative stress
}

\author{
Anneke C Blackburn ${ }^{1}$, Marjorie Coggan ${ }^{1}$, Alison J Shield ${ }^{1,2}$, Jean Cappello ${ }^{1}$, Angelo Theodoratos ${ }^{1}$, Tracy P Murray ${ }^{1}$, \\ Melissa Rooke ${ }^{1}$, Claire Z Larter $^{3}$, Mark E Koina ${ }^{4}$, Jane E Dahlstrom ${ }^{1,3,4}$, Klaus I Matthaei ${ }^{1}$ and Philip G Board ${ }^{1}$
}

Glutathione transferase kappa (GSTK1-1) is a highly conserved, mitochondrial enzyme potentially involved in redox reactions. GSTK1-1-deficient mice were generated to further study the enzyme's biological role. Reduced and total glutathione levels in liver and kidney were unchanged by GSTK1-1 deficiency and NADPH quinone oxidoreductase 1 expression was not elevated indicating that there is no general underlying oxidative stress in $G s t k 1^{-1-}$ mice. Electron microscopy of liver and kidney showed no changes in mitochondrial morphology with GSTK1-1 deficiency. The death of a number of $G s t k 1^{-/-}$males with urinary tract problems prompted close examination of the kidneys. Electron microscopy revealed glomerular basement membrane changes at 3 months, accompanied by detectable microalbuminuria in male mice (albumin:creatinine ratio of $2.66 \pm 0.83$ vs $1.13 \pm 0.20 \mathrm{mg} / \mathrm{mmol}$ for $G s t k 1^{-1-}$ and wild-type (WT), respectively, $P=0.001)$. This was followed by significant foot process effacement (40-55\% vs $10 \%$ for $G s t k 1^{-1-}$ and WT, respectively) at 6 months of age in all Gstk $1^{-1-}$ mice examined. Kidney tubules were ultrastructurally normal. Compared with human disease, the Gstk $1^{-1-}$ kidneys show changes seen in glomerulopathies causing nephrotic syndrome. Gstk ${ }^{-1-}$ mice may offer insights into the early development of glomerular nephropathies.

Laboratory Investigation (2011) 91, 1572-1583; doi:10.1038/labinvest.2011.107; published online 8 August 2011

KEYWORDS: GSTK1-1 deficiency; glutathione transferase kappa; glomerular nephropathy; knockout mice; mitochondria

In mammals, there are three structurally distinct families of proteins that catalyze glutathione transferase (GST) reactions. These families have been generally termed the cytosolic, microsomal and mitochondrial GSTs.

Although some of the cytosolic GSTs are also found within mitochondria, the kappa class GST is found specifically within mitochondria and peroxisomes. ${ }^{1}$ There is a single kappa class gene in humans, rats and mice and their amino acid sequences show little identity with the cytosolic or microsomal GST families. ${ }^{2,3}$ Although the structure of the kappa class GSTs contains a thioredoxin fold domain that is reminiscent of the cytosolic GSTs, ${ }^{3,4}$ it contains a polyhelical domain embedded in the linear sequence between the $\mathrm{N}$-terminal region $\beta$-strands. This contrasts with the cytosolic GSTs where the $\alpha$-helical domain is encoded entirely within the C-terminal sequence. This difference in structure suggests that the kappa class GSTs evolved via a distinct but convergent evolutionary path. The primary sequence and structure of the kappa class enzymes is more closely related to the prokaryotic disulphide-bond-forming oxidoreductase (DsbA) and 2-hydroxychromene-2-carboxylate isomerases than the mammalian cytosolic GSTs. ${ }^{3}$ Previous studies have shown that the kappa class GST is found in many tissues but is most highly expressed in heart, kidney, liver and skeletal muscle. ${ }^{5}$ Despite the knowledge of their structure and evidence of their capacity to conjugate glutathione to the classic xenobiotic substrate 1-chloro-2,4-dinitrobenzene, little is known about the role of kappa class GSTs in vivo. Its similarity to DsbA and location in peroxisomes and mitochondria where reactive oxygen species and peroxides are generated suggest that a role in a redox process is possible. Alternatively as the 2-hydroxychromene-2-carboxylate isomerases are involved in the bacterial metabolism of naphthalene, it is also possible that the kappa class GST is involved in xenobiotic detoxication reactions. ${ }^{6}$

To gain an understanding of the role of GST kappa (GSTK1-1) in vivo, we generated a Gstk1-deficient mouse. We report here the unexpected discovery that mice deficient in

\footnotetext{
${ }^{1}$ John Curtin School of Medical Research, Australian National University, Canberra, Australia; ${ }^{2}$ Faculty of Health, University of Canberra, Canberra, Australia; ${ }^{3}$ ANU Medical School, The Canberra Hospital, Canberra, Australia and ${ }^{4}$ Department of Anatomical Pathology, The Canberra Hospital, Canberra, Australia Correspondence: Professor PG Board, PhD, John Curtin School of Medical Research, Australian National University, PO Box 334, Canberra, ACT 2601, Australia. E-mail: Philip.Board@anu.edu.au 
GSTK1-1 displayed subclinical glomerular disease with alteration to the glomerular basement membranes (GBMs) preceding damage to the podocyte foot processes. Further study of the renal abnormality that we have discovered in Gstkl knockout mice may prove useful in elucidating the early mechanism and causes of nephropathy.

\section{MATERIALS AND METHODS}

\section{Generation and Genotyping of GSTK1-1-Deficient Mice}

The mouse Gstk1 gene spans about $4.3 \mathrm{~kb}$ on chromosome 6 and contains 8 exons. $^{2}$ A reverse transcribed pseudo gene occurs on chromosome 19. Gstk1 fragments containing exons 1-3 (1023 bp) and 6-8 (1841 bp) were amplified from $\mathrm{BALB} / \mathrm{c}$ genomic DNA by PCR. A targeting construct (pKOmGSTK1) that replaced exons 4 and 5 with a neomycin resistance gene was constructed in the plasmid pKO Scrambler (Stratagene) as shown in Figure 1. The gene was modified by homologous recombination ${ }^{7}$ in $\mathrm{BALB} / \mathrm{C}$ embryonic stem cells (ES) that were generously provided by Dr Birgit Ledermann (University of Zurich). The targeted ES cells were selected and implanted into C57BL/6 blastocysts as previously described. ${ }^{8}$ Male chimeric mice were crossed to $\mathrm{BALB} / \mathrm{c}$ females and resulting heterozygous $\left(G s t k 1^{+/-}\right)$mice were intercrossed to produce isogenic BALB/c Gstk1 ${ }^{-/-}$mice. Mice were maintained in micro-isolator cages in a specific pathogen-free mouse breeding facility, with controlled temperature and a $12 \mathrm{~h}$ light-dark cycle. All studies were undertaken with the approval of the Australian National University Animal Ethics Experimentation Committee under the guidelines established by the Australian National Health and Medical Research Council.

Mice were genotyped by PCR analysis of genomic DNA obtained from tail samples. The oligonucleotide primer pairs MK5F/mkappa R and JLneoA/mkappa R (Figure 1) were designed to amplify DNA from wild-type (WT) and knockout genes. Gstk1 $1^{+1+}$ mice gave rise to a single 800 -bp amplicon, Gstk $1^{-1-}$ mice gave a single 1092-bp amplicon, and Gstk1 ${ }^{+/-}$mice showed both the 800-bp and 1092-bp amplicons after PCR analysis (Figure 1).

\section{Western Blotting}

Protein extracts from a range of frozen mouse tissues were prepared by homogenizing tissues in mitochondrial homogenizing buffer consisting of $250 \mathrm{mM}$ sucrose, $1 \mathrm{mM}$ EDTA and $10 \mathrm{mM}$ HEPES pH 7.4 (approximately $250 \mathrm{mg}$ tissue with $750 \mu \mathrm{l}$ buffer) with an IKA-Ultra-Turrux T25 homogenizer. Homogenates were centrifuged for $20 \mathrm{~min}$ at $8000 \times g$ at $4{ }^{\circ} \mathrm{C}$, and $100 \mu \mathrm{g}$ of protein from the supernatant used in western blotting as described previously. ${ }^{8}$ Antisera raised against rat $\mathrm{NAD}(\mathrm{P}) \mathrm{H}$ :quinone oxidoreductase 1 (NQO1), mGSTA1/2, mGSTA3-3, rGSTA4-4, rGSTM1-1, hGSTM3-3, mGSTP1/2 and mGSTK1-1 was generously provided by Professor John Hayes (University of Dundee). Antisera against recombinant hGSTK1-1, hGSTZ1-1, mGSTT1-1 and mGSTO1-1 were raised in rabbits in the

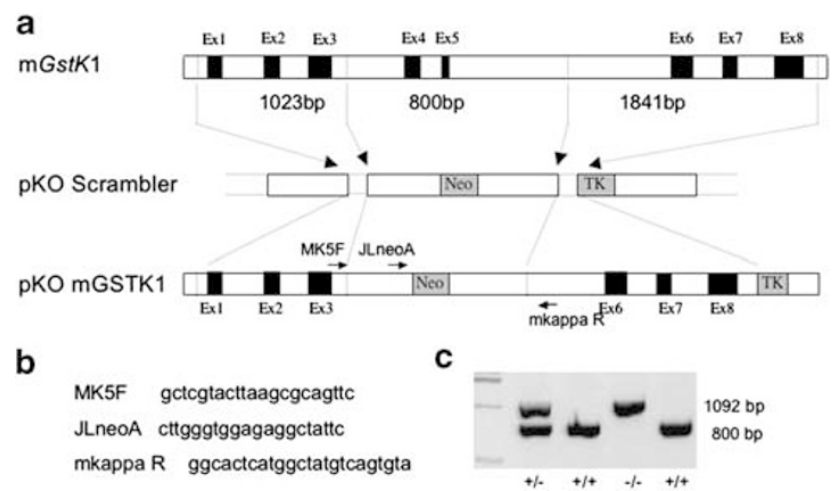

Figure 1 Gstk1 gene knockout construct. Panel (a) shows the organization of the BALB/c mouse Gstk1 gene and the DNA construct made in pKO Scrambler to inactivate the gene in BALB/c ES cells. The relative positions of the genotyping primers are shown in panel (a) and their sequence is listed in panel (b). Panel (c) shows a typical PCR genotyping experiment.

laboratory of Professor Philip Board (Australian National University, Canberra, Australia).

\section{Enzyme-Activity and Glutathione Measurements}

Total enzymatic activity with 1-chloro-2,4-dinitrobenzene (CDNB) as a substrate was determined spectrophotometrically ${ }^{9,10}$ on protein extracts prepared from frozen tissues as for western blotting. For mitochondrial GST activity, fresh tissues were homogenized in mitochondrial homogenizing buffer. Debri and nuclei were removed by centrifugation twice at $1000 \times g$ for $10 \mathrm{~min}$ at $4{ }^{\circ} \mathrm{C}$. Heavy mitochondria were then pelleted by centrifugation at $3000 \times g$ for $10 \mathrm{~min}$ at $4{ }^{\circ} \mathrm{C}$, and washed once with mitochondria homogenizing buffer. Western blotting for cytochrome C and GSTP1 verified the enrichment of mitochondria with minimal cytoplasmic carry over in these preparations (data not shown). Total glutathione (reduced glutathione (GSH) and glutathione disulfide (GSSG)) and GSSG concentrations in mouse tissues were assayed with the glutathione reductase cycling assay as described previously. ${ }^{11,12}$

\section{Pathology and Electron Microscopy}

Tissues were fixed in neutral buffered formalin, then processed and embedded in paraffin. Sections $4 \mu \mathrm{m}$ thick were stained with hematoxylin and eosin for pathologic assessment. Kidney sections were also stained with Periodic Acid Schiffs (PAS) stain or Periodic Acid Silver Methenamine (PASM) stain for basement membrane changes.

For electron microscopic (EM) examination, samples of kidney and liver were collected fresh, fixed and processed as described previously. ${ }^{8}$ Foot process effacement was evaluated according to Jo et al..$^{13}$ A minimum of 15 glomeruli per group were assessed for the degree of foot process effacement, with the mean \pm s.d. for each genotype presented, and significance was tested by $t$-test. The presence of morphological changes to the GBM was assessed for each 
animal and graded between 0 and 3. The thickness of the GBM was measured in both uninvolved areas (200 measurements per group) and involved areas (100 measurements per group).

\section{Analysis of Mouse Blood and Urine Chemistry}

For hematological analysis, approximately $200 \mu \mathrm{l}$ of blood was collected onto dried heparin and analyzed on a Advia 2120 hematology analyzer (Siemens Victoria) that had been specifically calibrated for BALB/c mouse blood cells.

Urine from male and female mice was screened for standard urine indicators using Multistix Reagent Strips (Bayer). Urine and serum albumin and creatinine, plasma cholesterol, triglycerides and ultra-high-density lipoprotein levels were determined by ACT Pathology (Canberra, Australia) using Abbott Clinical Chemistry on the ARCHITECT c8000 system (Abbott Laboratories, Botany, NSW). Mouse urine was collected by massaging the abdomen of mice, and collecting urine in a Petri dish. Urine samples collected over several days were pooled to allow for undiluted analysis on standard chemistry analyzers. Plasma for lipid analysis was collected from retro-orbital bleeding of mice $(\sim 200 \mu \mathrm{l}$ blood) into EDTA.

Reducing SDS-PAGE analysis of male mouse urine proteins was performed on $8-25 \%$ gradient PhastGels (Pharmacia, GE Healthcare) after diluting 1 in 10 with water, followed by Coomassie-blue staining.

\section{Immunohistochemistry}

The presence of immune deposits in the kidneys was examined by direct immunofluorescence on tissues snap frozen in liquid nitrogen. Sections $4 \mu \mathrm{m}$ thick were cut and stained with FITC conjugated antibodies (sheep anti-human, cross reacting with mouse, Millipore) against IgM, IgG, C3 and IgA and photographed using a Zeis AxioCam Hrc camera attached to an axioskop 2plus microscope. The lack of IgG staining was confirmed using an anti-mouse IgG antibody from BD Biosciences.

Immunohistochemistry was performed on the Bond automated system (Vision Biosystem), following a standard protocol. Briefly, 2 and $4 \mu \mathrm{m}$ formalin-fixed, paraffin-embedded tissues sections were dewaxed, rehydrated through graded alcohol and stained with antibodies to collagen IV (2150-1470, AbD Serotec, Oxford, UK, dilution 1/500) and laminin EHS (L9393, Sigma-Aldrich, MO, USA, dilution 1/500). We used heat retrieval for $28 \mathrm{~min}$ at a $\mathrm{pH} 6$.

\section{Dexamethasone Treatment}

Male mice (five WT and six Gstk1 ${ }^{-/-}$) were administered $1 \mathrm{mg} / \mathrm{kg}$ dexamethasone (DBL Dexamethasone sodium phosphate for injection) by i.p. injection 5 days per week. Injections started at 17 weeks of age and continued until 26 weeks of age, when mice were killed and tissues collected for light and electron microscopy.

\section{Adiponectin ELISA}

Total serum adiponectin was measured using a commercial ELISA according to the manufacturer's instructions ( $\mathrm{R}$ and $\mathrm{D}$ systems, Minneapolis, MN, USA).

\section{RESULTS}

\section{Growth and Development of GSTK1-Deficient Mice}

The Gstk1 $1^{-/}$mice bred successfully and were without any obvious phenotype. The offspring from heterozygous crosses were in the expected Mendelian ratios for both males and females, and no significant differences were observed in mouse body weight from weaning through to 18 months of age between WT and Gstk1 ${ }^{-1-}$ mice of the same gender.

The tissues of WT and Gstk1 ${ }^{-1-}$ mice were examined for expression of GSTK1-1 by western blotting. GSTK1-1 was expressed in many different tissues, with high levels in heart, testis, lung, liver and kidney (Figures $2 \mathrm{a}$ and $\mathrm{b}$ ) and very low expression in the spleen (Figures $2 \mathrm{a}$ and $\mathrm{c}$ ), consistent with previous reports. ${ }^{5}$ To confirm the successful inactivation of Gstk1, western blots of liver, kidney and heart from Gstk1 ${ }^{-1-}$ mice were examined (Figures $2 \mathrm{c}$ and $\mathrm{d}$ ) and no GSTK1-1 protein could be detected. A specific substrate that can detect GSTK1-1 activity in the presence of other GSTs has not been identified, however, mouse GSTK1-1 has relatively high activity toward the general GST substrate CDNB. ${ }^{2}$ The deletion of the Gstk1 gene had no significant impact on the total

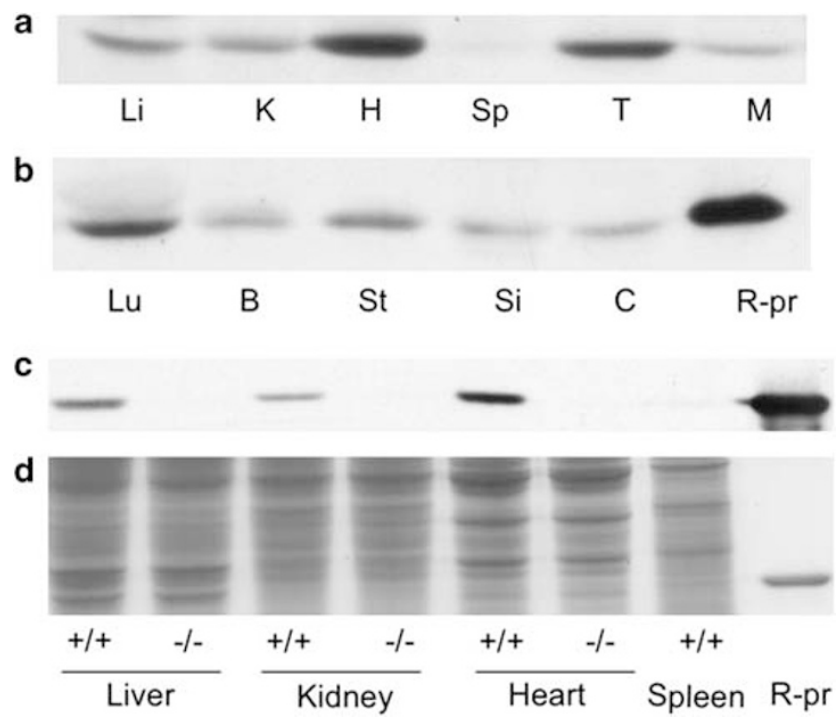

Figure 2 Western blots of GSTK1-1 in tissues from Gstk $1^{+1+}$ and Gstk $1^{-1-}$ mice. Panels (a) and (b) show tissues from WT Gstk $1^{+/+}$mice; liver (Li), kidney $(K)$, heart $(H)$, spleen $(\mathrm{Sp})$, Testis $(\mathrm{T})$, skeletal muscle $(\mathrm{M})$, lung $(\mathrm{Lu})$, brain (B), stomach (St), small intestine (Si), colon (C) and recombinant mGSTK1-1 (R-pr). The mice were 3-4 months old and $100 \mu \mathrm{g}$ of tissue protein was added to each track. Panel (c) shows GSTK1-1 in tissues from Gstk $1^{+/+}$and Gstk $1^{-/-}$mice. The tissue source and the genotypes of the samples in (c) and (d) are shown below panel (d). Panel (d) is a loading control and shows a Coomassie-blue stained gel that is a duplicate of the gel blotted in panel (c). 

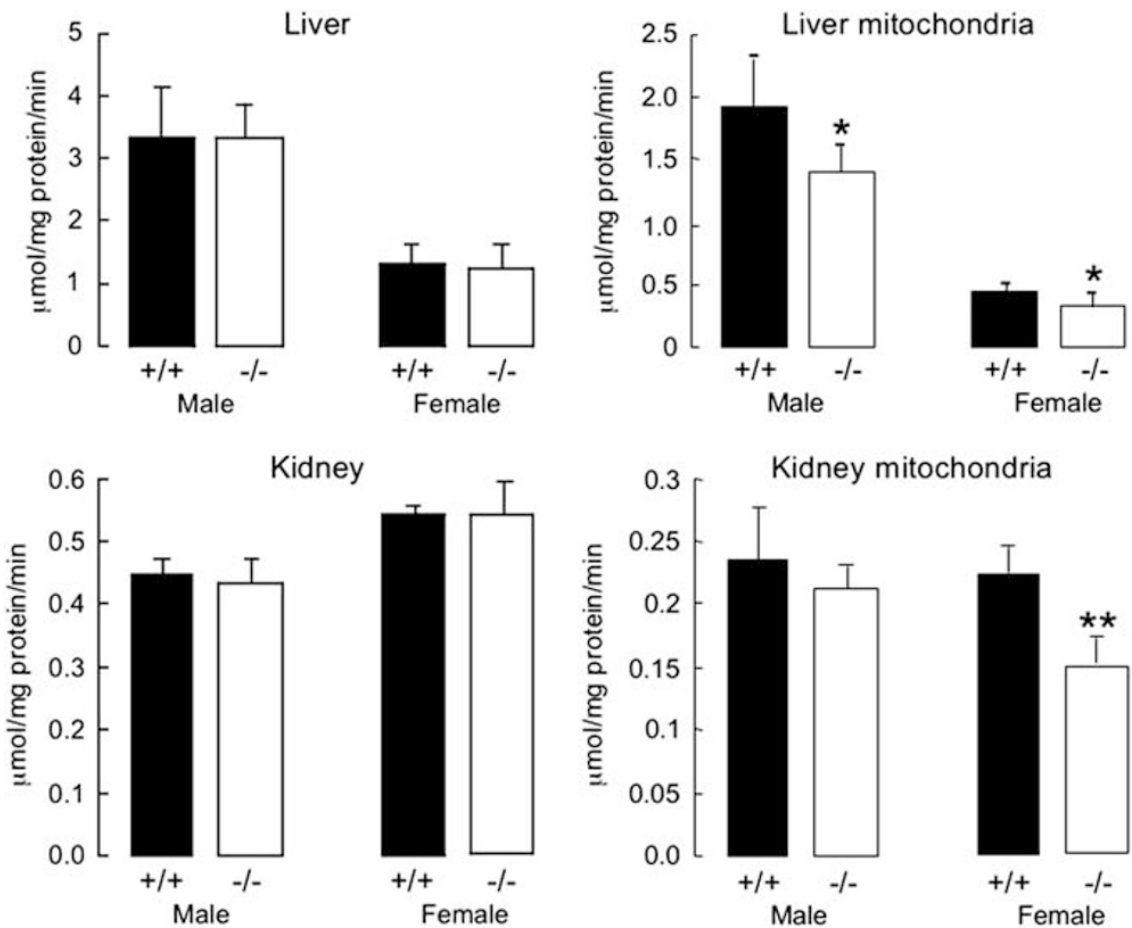

Figure 3 The effect of GSTK1-1 deficiency on CDNB conjugating activity in whole tissue homogenates and in mitochondria. Activity was determined in the liver and kidney of 11-week-old mice ( $n=4-6$ mice per group. Mean \pm s.d., ${ }^{\star} P=0.05,{ }^{*} P=0.001$ ).

CDNB conjugating activity of the liver or kidney (Figure 3). (The large difference in activity in the liver between males and females reflects the significantly higher expression of hepatic GSTP1-1 in males that has been previously described). ${ }^{12,14}$ This also suggests that there is no increase in expression of other GST classes to compensate for deficiency of GSTK1-1. This was investigated further by western blotting of liver and kidney tissues for specific GST classes. No differences in expression levels of GSTZ1-1, GSTP1-1/2-2, GSTM1-1, GSTT1-1, GSTA1-1/2-2, GSTA3-3, GSTA4-4 and GSTO1-1 were observed between WT and $G s t k 1^{-1-}$ mice (data not shown). As GSTK1-1 is localized to the mitochondria, CDNB activity of mitochondrial preparations from liver and kidney were measured. In most cases, a 25-30\% decrease in CDNB activity was found, indicating GSTK1-1 is a significant contributor to mitochondrial GSH conjugating capacity (Figure 3 ).

Although other GST classes do not appear to be compensating for GSTK1-1 deficiency, it is notable that tissues from heterozygous mice $\left(G s t k 1^{+/-}\right)$mice showed similar levels of GSTK1-1 protein to WT mice (data not shown), suggesting that expression is elevated from the single gene copy in the heterozygous mice to maintain GSTK1-1 protein at WT levels. It is also possible that Gstk1 expression occurs from only one chromosome but this seems unlikely. This result is in contrast to Gstz1 ${ }^{+/-}$and $\mathrm{Gsta4}^{+/-}$mice, where protein expression levels were intermediate between $-/-$ and WT levels demonstrating haploinsufficiency. ${ }^{8,15}$

\section{Oxidative Stress is Not Evident in Gstk1 ${ }^{-I-}$ Mice}

As GSTK1-1 has been suggested to protect against oxidative stress, several characteristic markers of oxidative stress were evaluated in the tissues of Gstk1 ${ }^{-1-}$ mice. Total glutathione levels (GSH + GSSG) were examined in liver and kidney of 11-week-old Gstk1 ${ }^{-1-}$ mice, and were found to be unchanged compared with WT (Figure 4). Although male kidney GSSG appeared to be slightly elevated in Gstk $1^{-/-}$mice, this was not replicated in a second independent set of five mice of a similar age. Similar results were obtained for female liver and kidney (data not shown).

$\mathrm{NAD}(\mathrm{P}) \mathrm{H}$ :quinone oxidoreductase 1 (NQO1) expression is elevated when cells are subjected to oxidative stress. There was no difference in NQO1 expression in liver, kidney, (Figure 5) heart, lung and testes (data not shown) between WT and Gstk1 $1^{-\prime-}$ mice, indicating that little or no oxidative stress is generated by GSTK1-1 deficiency.

\section{Organ Weights are Altered by GSTK1 Deficiency}

The weights of major organs were examined in WT and $G s t k 1^{-1}$ mice as a gross indicator of possible pathologies. Data were collected from male and female 3-month-old mice and two sets of 8-month-old mice, with the data from the larger set of 8-month-old mice shown in Table 1. Consistent changes in the weight of kidneys, spleen and testes were noted in all sets of mice although the size and level of significance of the change varied (indicated in brackets). Kidneys were increased (6-11\%), spleens were decreased 

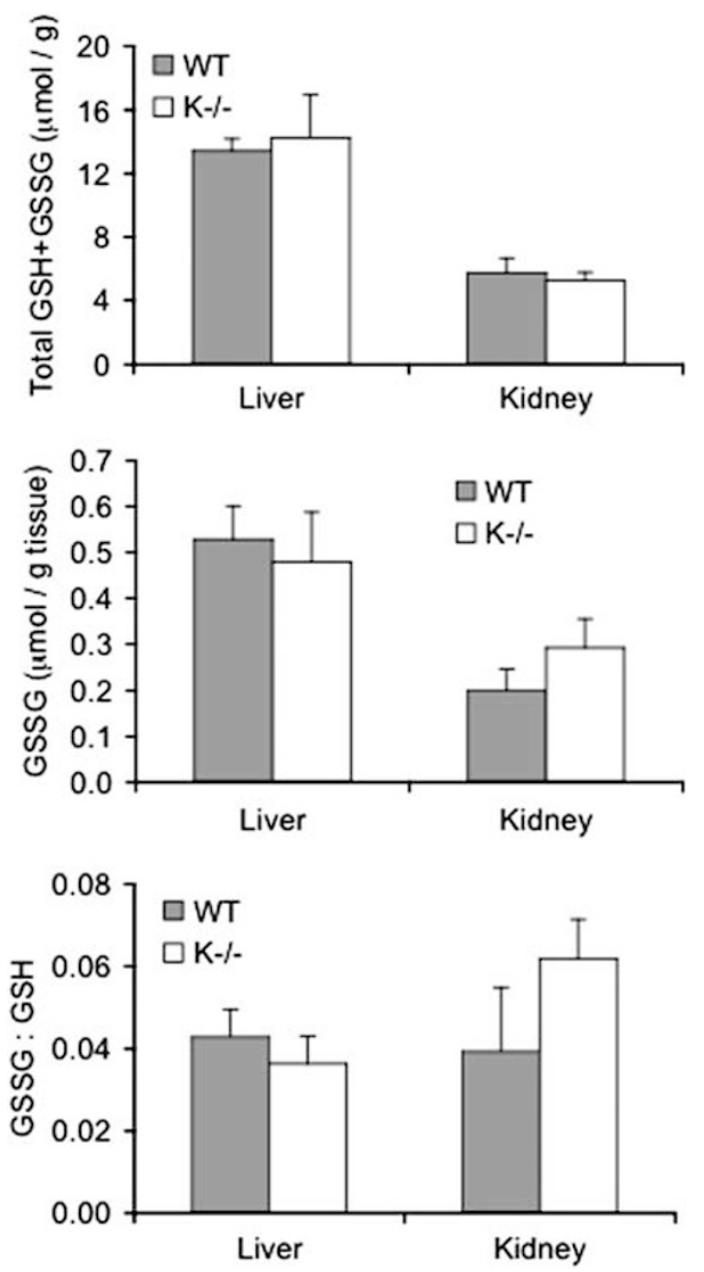

Figure 4 Total glutathione, oxidized glutathione (GSSG) and the GSH:GSSG ratio in liver and kidney. Mice were 11-week-old males. ( $n=4-6$ per group. Mean \pm s.d.).

a Liver

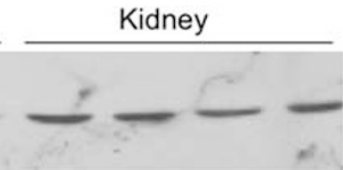

b

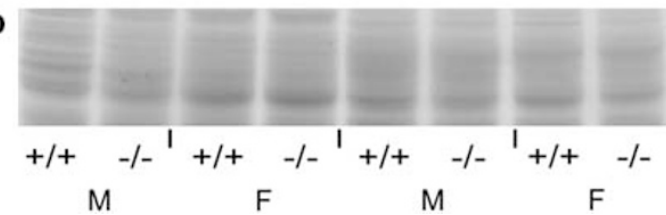

Figure 5 NQO1 expression in the liver and kidney of $\mathrm{WT}\left({ }^{+/+}\right)$, and Gstk $1^{-/-}$mice. Panel (a) shows a western blot of NQO1. Panel (b) is a loading control and shows a Coomassie-blue stained gel that is a duplicate of the gel blotted in panel (a). The mice were 3-4 months old and $100 \mu \mathrm{g}$ of tissue protein was added to each track. The genotypes and gender of the samples are shown below panel (b).

(6-52\%) and testes were increased (9-14\%) in weight. In contrast, total body weights and liver weights were unchanged by GSTK1-1 deficiency.

\section{Hematological Analysis, Blood Lipids and General Pathology of GSTK1-1-Deficient Mice}

As a result of the differences in spleen weights, the histology of the spleens was examined. No significant differences were noted in the spleens showing smaller differences in weight (eg, $21 \%$ decrease), however, in the male mice displaying a $52 \%$ decrease in spleen weight, a notable reduction in red pulp was observed (data not shown). This prompted investigation of the hematological profile of 15-week-old male mice. Although there was a trend toward decreased numbers of neutrophils and monocytes, there were no significant differences in red or white cell counts or in the differential white cell count between WT and Gstk1 ${ }^{-1-}$ mice.

Given the expression of GSTK1-1 protein in liver mitochondria and peroxisomes ${ }^{1}$ and the importance of these organelles in lipid homeostasis, we investigated circulating lipid levels in WT and Gstk1 ${ }^{-1-}$ mice receiving normal mouse chow. A $16 \%$ decrease in triglyceride levels was noted in 25 -week-old male mice $(1.17 \pm 0.21$ vs $0.98 \pm 0.15 \mathrm{mmol} / \mathrm{l}$ for WT and $G s t k 1^{-1-}$ mice, respectively, $n=10$ per group, $P=0.046$ ), however, this decrease was a not maintained in older cohorts of mice. No trends were noted for cholesterol or high-density lipoproteins.

The liver, kidney, lungs, heart, thymus, adrenal gland, intestinal tract, lymph nodes, skeletal muscle and testes of 11-week-old healthy male and female WT and Gstk1 $1^{-1-}$ mice were examined by light microscopy. No gross or microscopic pathological differences were observed between the WT and Gstk1 ${ }^{-1-}$ groups for each gender. As GSTK1-1 has been found in mitochondria, we examined mitochondrial morphology by EM. No changes in mitochondrial morphology were found in the livers of 15 months old Gstk $1^{-1-}$ mice or in kidneys from 3- to 15-month-old mice.

\section{Survival of GSTK1 ${ }^{-1-}$ Mice}

WT, Gstk1 ${ }^{+/-}$and Gstk1 ${ }^{-/-}$mice were monitored for general health problems until at least 18 months of age. In females, there were no differences in survival between WT and $G s t k 1^{-1-}$ mice over this period, and no significant occurrence of any unusual tumors/pathologies in Gstk1 ${ }^{-/-}$ mice (Figure 6a). In males, however, the survival curves appeared different in shape, crossing over at approximately 500 days (Figure 6b). Examination of the necropsy information found two major likely causes of death or killingkidney/urinary tract pathologies occurring from around 6 months of age and occurring more frequently in Gstk1 ${ }^{-1-}$ mice, and heart pathologies occurring more frequently in WT mice over 1-year old. The heart pathology was consistently a large thrombus in the left atrium, often with calcification, causing complete obstruction of the blood flow, similar to that observed previously in BALB/c-Gstz1-deficient mice, ${ }^{8}$ and was observed upon necropsy in $9 / 28$ (32\%), 7/36 (19\%) and $3 / 20(15 \%)$ of Gstk $1+/+,+/-$ and $-/-$ male mice, respectively, with the survival curves differing significantly (Figure $6 c, P=0.028$, log-rank test). The kidney pathologies 
Table 1 Organ weight as a percentage of body weight in wild-type and Gstk $1^{-/-}$mice (mean \pm s.d.)

\begin{tabular}{|c|c|c|c|c|c|c|}
\hline & $N$ & Body wt (g) & Liver (\% body wt) & Kidney (\% body wt) & Spleen (\% body wt) & Testes (\% body wt) \\
\hline \multicolumn{7}{|l|}{ Males } \\
\hline Gstk $1^{-/-}$ & 7 & $28.8 \pm 3.6$ & $5.20 \pm 0.54$ & $2.15 \pm 0.17$ & $0.24 \pm 0.03$ & $0.40 \pm 0.02$ \\
\hline$\%$ change & & $-1.3 \%$ & $-8.0 \%$ & $9.9 \%$ & $-52.2 \%$ & $14.0 \%$ \\
\hline \multicolumn{7}{|l|}{ Females } \\
\hline Wild type & 10 & $25.4 \pm 3.6$ & $4.78 \pm 0.23$ & $1.23 \pm 0.03$ & $0.46 \pm 0.03$ & \\
\hline Gstk1 $1^{-/-}$ & 10 & $26.0 \pm 2.6$ & $4.88 \pm 0.19$ & $1.37 \pm 0.03$ & $0.36 \pm 0.04$ & \\
\hline$\%$ change & & $2.1 \%$ & $2.1 \%$ & $11.1 \%$ & $-21.4 \%$ & \\
\hline
\end{tabular}
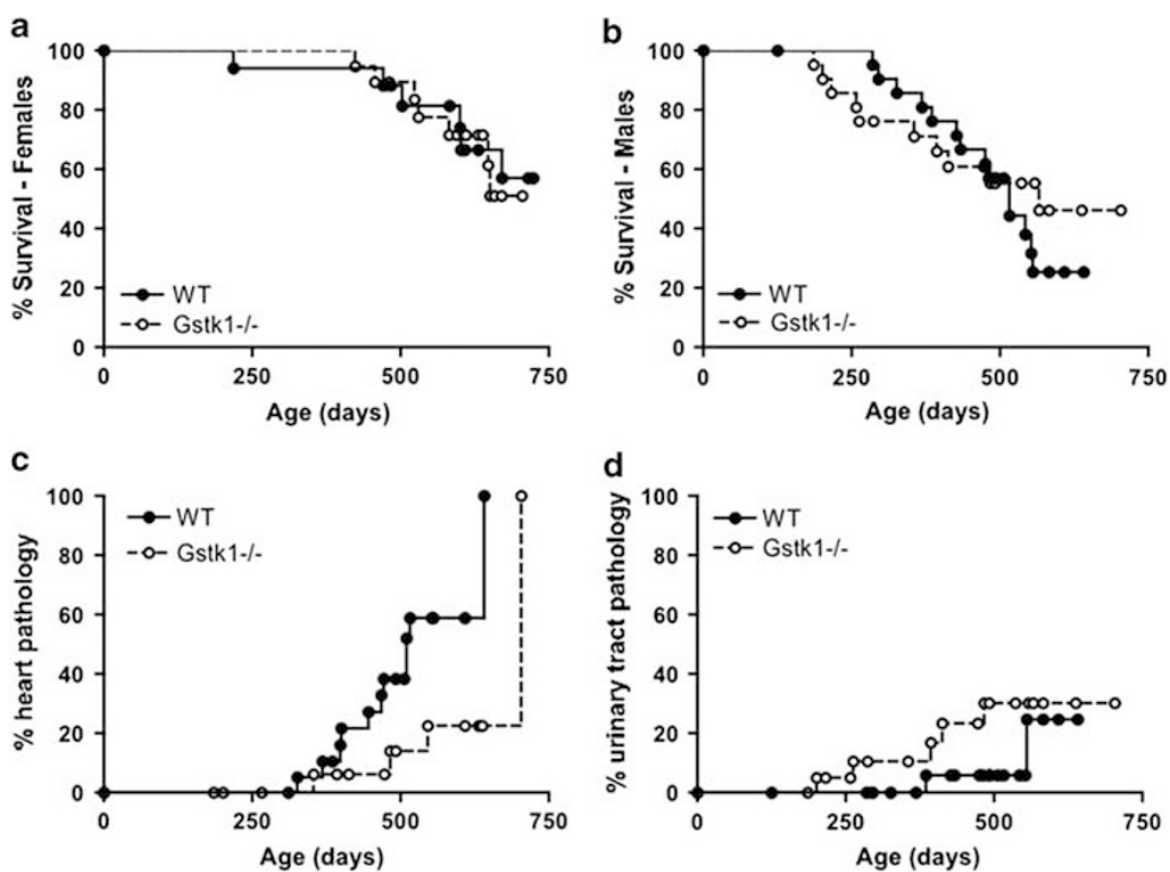

Figure 6 Kaplan-Meier event plots for WT and Gstk $1^{-/-}$mice. The survival curve for all causes of death/killing are shown for females in panel (a) and males in panel (b). Panel (c) shows death from thrombus in the heart of male mice $(P=0.028$, log-rank test), and panel (d) shows death from kidney/urinary tract pathologies in male mice $n=17$ and 19 for female mice and $n=22$ and 21 for male mice. WT $(\bullet)$ and Gstk $1^{-/-}(O)$.

were evident much earlier, when a number of male Gstk1 ${ }^{-1-}$ mice died suddenly between 29 and 38 weeks of age, and upon necropsy, were found to have very large distended bladders with blood evident in the urine. Histological examination revealed blockages of the urinary tract, with blood and acute inflammation in the bladder and urethra. Gram staining revealed the presence of Escherichia coli within the tissues of some mice. The kidney/urinary tract pathologies occurred in 2/28 (7\%), 7/36 (19\%) and 5/20 (25\%) of Gst1k+/+, + /- and -/- male mice, respectively, however, the survival curves were not significantly different (Figure 6d).

\section{Glomerular Nephropathy Occurs in Gstk $1^{-I-}$ Mice}

Closer examination of the urinary tract of healthy 12-weekold Gstk1 $1^{-/-}$mice found no evidence of kidney disease by light microscopy or routine (dipstick) protein and blood detection in the urine. There was no evidence of an inflammatory infiltrate or scaring in kidneys or bladders, suggesting chronic infection was not a contributing factor to the blockages observed in the ageing mice. Hydronephrosis was also not observed in the absence of obstruction. However, electron microscopy on 21-week-old males detected GBM changes and abnormally high amounts of foot process 

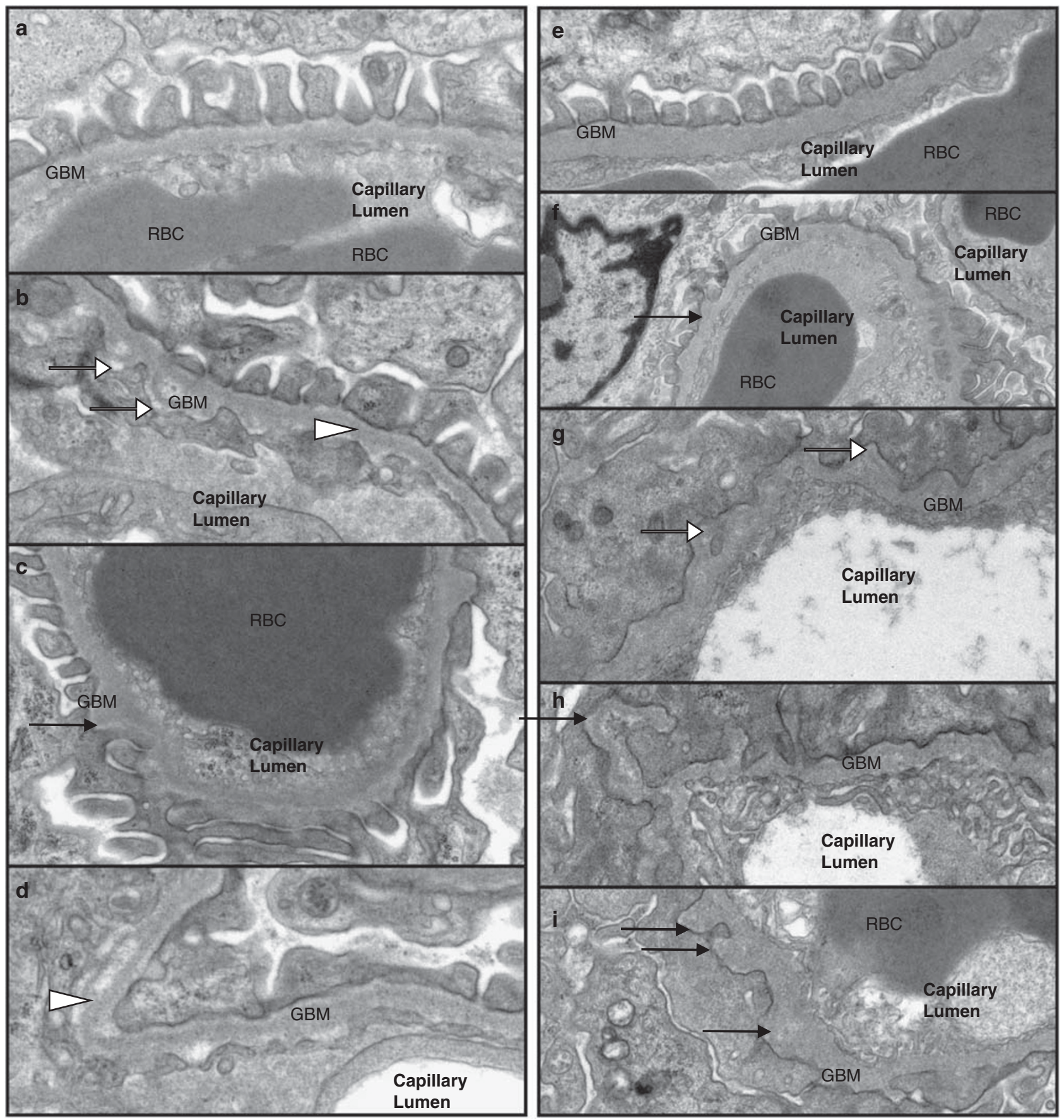

Figure 7 Electron micrographs of kidney glomeruli. WT mice at 3 months (a) and 6 months (e) show normal GBM and foot process structure. Kidneys from $G s t k 1^{-1-}$ mice at 3 months (b-d) of age show GBM changes including projections (filled arrows), splits (open arrows) and laminations (open arrow heads). Foot processes are still within normal ranges. Kidneys from Gstk $1^{-1-}$ mice at 6 months of age (f-i) show much more severe GBM changes, with no normal foot processes being evident (ie, complete effacement) in the areas included in images (g-i).

effacement in $G s t k 1^{-1-}$ mice compared with age-matched WT mice (Figure 7). Ultrastructural changes in the glomerulus were subsequently evaluated at different ages and are summarized in Table 2. At 6 weeks of age, Gstk1 $1^{-1-}$ glomeruli showed no abnormal changes ( $n=2$ males). EM at 3 months of age revealed GBM changes occurring, including lamina- tions, splits, subepithelial protrusions (similar to 'spikes' in membranous glomerular nephritis stage II) and lucent areas (Figures $7 \mathrm{~b}-\mathrm{d}$ ). Expanded mesangial matrix was also noted. Both male and female Gstk1 $1^{-1-}$ mice displayed the basement membrane changes at 3 months of age, but no increase in foot process effacement was evident at this age 
Table 2 Changes in glomerular basement membrane ultrastructure, foot process effacement and immune deposits with age

Age $\quad 3$ mo $\quad 15$ mo

Males

Glomerular basement membrane: morphological changes (0-3)

Glomerular basement membrane: thickness (nm) (mean (s.d.))

Foot process effacement: \% (mean (s.d.))

$\begin{array}{ccccc} & \text { Wild type } & 18.1(5.7) & 10(3.2) & 23.4(12.9) \\ \text { Gstk1 } 1^{-/-} & 23.7(6.2)^{*} & 47.5(5.8)^{* *} & 39.7(4.3)^{* *} \\ n & \text { Wild type } & 5 & 7 & 2 \\ \text { Gstk1 } 1^{-/-} & 5 & 7 & 2\end{array}$

Females

Glomerular basement membrane: morphological changes (0-3)

\section{Wild type}

Gstk $1^{-1-}$

Glomerular basement membrane: thickness (nm) (mean (s.d.))

Foot process effacement: \% (mean (s.d.))

\begin{tabular}{|c|c|c|c|}
\hline Wild type & $105.4(6.6)$ & $107.6(7.5)$ & $111.0(8.2)$ \\
\hline Gstk1 $1^{-1-N O}$ & $105.8(8.7)$ & $107.6(7.5)$ & $111.9(8.2)$ \\
\hline Gstk1 $1^{-/-} A B$ & $217.4(23.5)$ & $231.6(20.9)$ & $239.6(24.0)$ \\
\hline Wild type & $17.8(5.5)$ & $10.0(4.1)$ & $37.8(2.6)$ \\
\hline Gstk $1^{-/-}$ & $23.4(6.0)^{*}$ & $39.7(4.3)^{* *}$ & $29.7(4.3)^{* *}$ \\
\hline Wild type & 5 & 5 & 2 \\
\hline Gstk1 $1^{-/-}$ & 5 & 5 & 2 \\
\hline
\end{tabular}

Immune deposits (C3, IgM) — males and females

Electron microscopy

Wild type

Gstk $1^{-1-}$

Immunofluorescence
Wild type

Gstk1 $1^{-1-}$
$110.0(7.0)$
$111.3(7.2)$
$223.5(21.4)$

$111.2(7.1)$

$112.3(7.6)$

$240.1(17.1)$

$116.3(7.5)$

$239.2(18.2)$

$.4(12.9)$

$(+)$

$++$

2

Abbreviations: GSTK1 ${ }^{-1-}$ NO, normal appearing areas of GBM; GSTK1 ${ }^{-1-}$ AB , abnormal areas of GBM; mo, months; ND, not determined.

${ }^{*} P<0.01$.

${ }^{*} P<0.0001$ compared with wild type.

(Table 2). The basement membrane changes persisted at 5-6 months of age, although they were of lower grade in females than in males. Significantly more foot process effacement was observed in both male and female Gstk1 ${ }^{-1-}$ mice compared with WT mice at 5-6 months of age (Figures $7 \mathrm{f}-\mathrm{i}$, Table 2). In areas with abnormal ultrastructure, the GBM of both male and female Gstk $1^{-/-}$mice were on average twice as thick at all ages investigated. This difference in thickening of the basement membrane was not evident by light microscopy with PAS or PASM staining of kidney sections (data not shown). Immunohistochemistry for components of the GBM found no obvious difference in the intensity of staining for collagen IV and laminin in Gstk1 ${ }^{-/-}$ mice (Figure 8). 

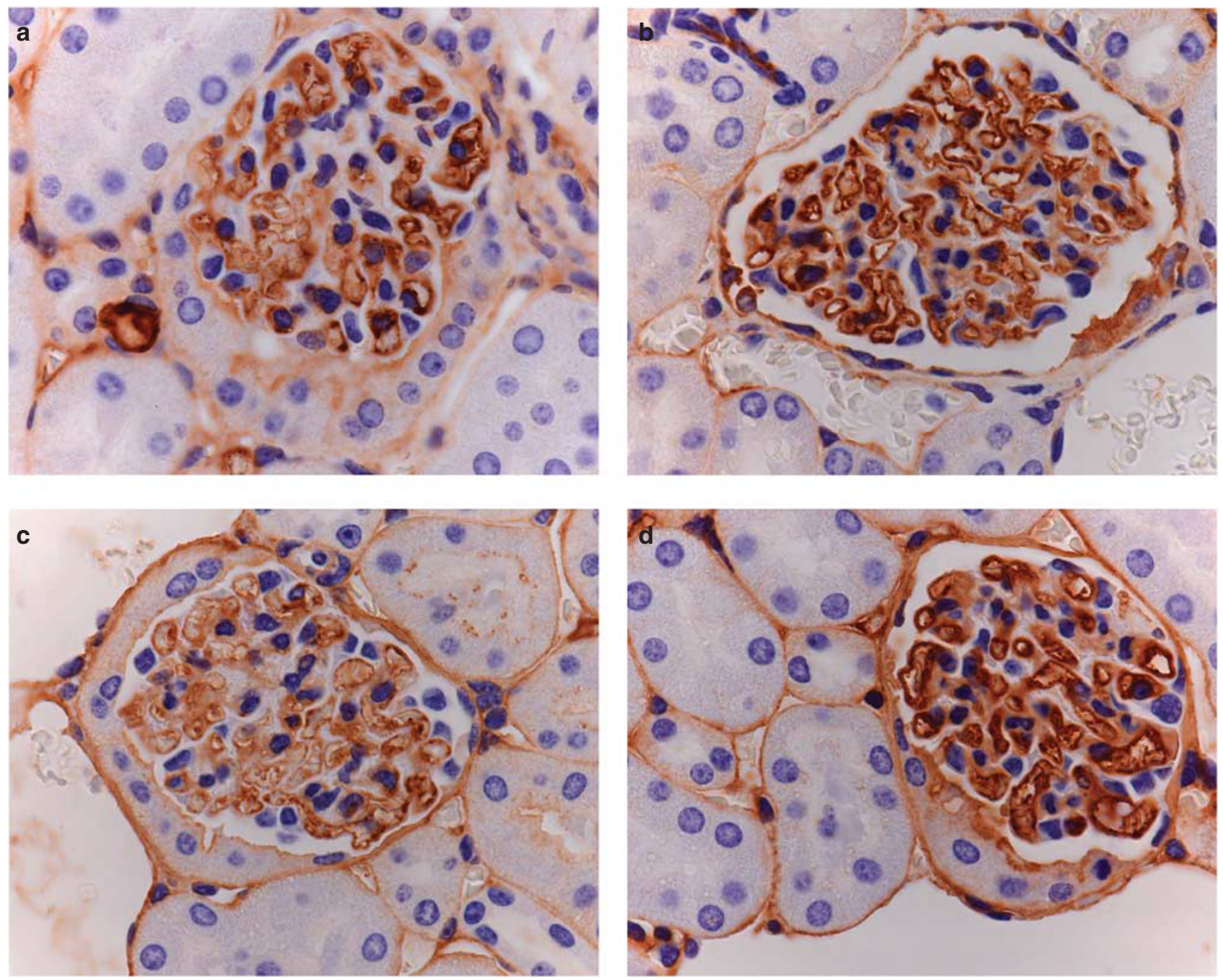

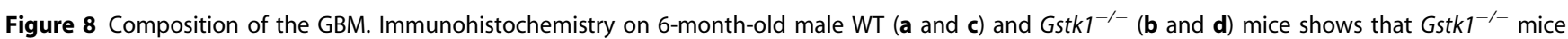
display no obvious difference in staining intensity for laminin ( $\mathbf{a}$ and $\mathbf{b}$ ) and collagen IV (c and $\mathbf{d}$ ) proteins in the GBM. (Photographed from $2 \mu \mathrm{m}$ sections at $\times 1000$ under oil immersion).

To determine whether oxidative stress in the kidney could have increased with age, and hence contributed to the ultrastructural changes observed, the measurement of total glutathione levels in kidneys was repeated on tissue from 6-month-old mice, however, there was still no difference in kidney total GSH + GSSG levels in male or female mice (data not shown). By 15 months, glomerulonephritis had developed to a similar extent in both WT and Gstk1 ${ }^{-/-}$mice, as is characteristic of the BALB/c strain, ${ }^{16}$ thus obscuring whether the phenotype associated with GSTK deficiency developed into more serious kidney disease. In contrast to the glomeruli, no abnormalities in the ultrastructure or morphology of renal tubules and tubular basement membranes were found by electron or light microscopy. In particular, the tubule mitochondria, where GSTK1 is reported to be expressed ${ }^{5}$ appeared normal in the Gstk1 $1^{-1-}$ mice.

The pathological changes observed in Gstk1 ${ }^{-1-}$ mice by EM were associated with microalbuminuria. Biochemical measurement of urinary albumin and creatinine was possible after pooling samples of urine collected over several days from individual mice. Although changes in total protein levels were not evident, urine albumin measurements revealed statistically significant microalbuminuria as indicated by elevated albumin/creatinine ratios in male Gstk1 $1^{-1}$ mice compared with WT counterparts at 12 weeks (Figure 9a, $P=0.002)$, and 26 weeks of age $(P=0.001)$. This was confirmed by SDS-PAGE analysis of urines from 7-month-old male mice (Figure 9b). By 45-48 weeks of age, both WT and Gstk1 ${ }^{-1-}$ mice displayed microalbuminuria and were no longer significantly different (Figure 9a). Albumin in female urine was consistently below the level of detection, and therefore any subtle changes could not be detected. Chronic microalbuminuria at this level did not significantly alter plasma albumin:creatinine ratios in Gstk1 $1^{-/-}$mice at any age (14-70 weeks old tested, data not shown). 

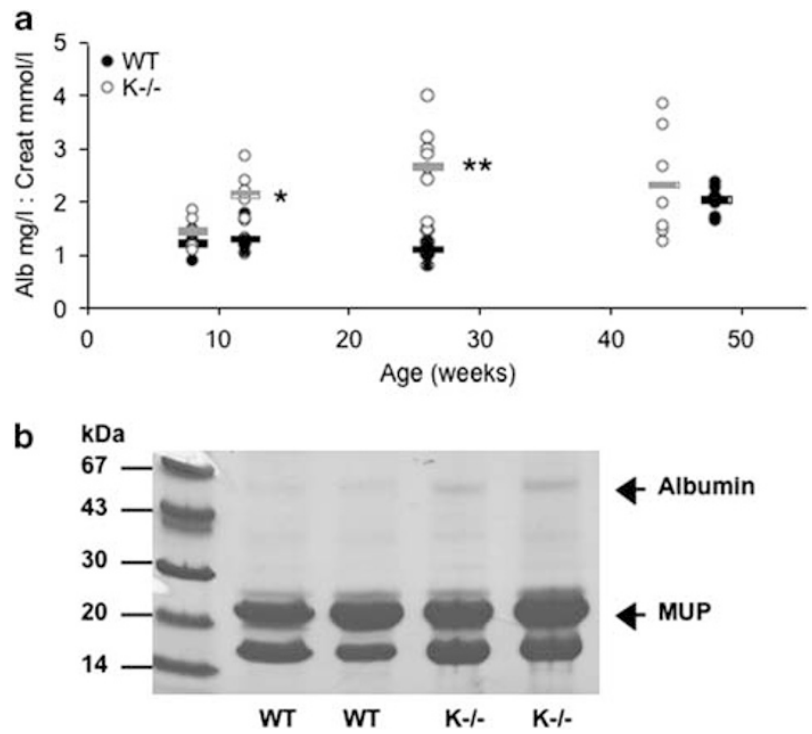

Figure 9 Urine albumin:creatinine ratio in male mice at different ages. (a) Significant microalbuminuria occurs in $G s t k 1^{-1-}$ mice compared with WT of the same age. The mean of each group is indicated by a horizontal bar. ${ }^{*} P=0.002$ and ${ }^{*} P=0.001$. (b) SDS-PAGE analysis of male mouse urine demonstrating selective increase in albumin content in the $G s t k 1^{-/-}$mice.

The glomerulonephritis of $\mathrm{BALB} / \mathrm{c}$ mice is characteristically accompanied by immune complex deposition. ${ }^{16} \mathrm{EM}$ showed mesangial protein deposits to be present in the kidneys of both WT and Gstk1 ${ }^{-/-}$mice, and the amount of these deposits increased with age (Table 2). Immunofluorescent staining confirmed that these deposits were immune complexes, primarily containing IgM staining with some C3 staining but no IgA or IgG. Notably, no difference was observed in the intensity of immune deposits seen by EM or by immunofluorescence between the WT and $G s t k 1^{-/-}$ kidneys at any age (Table 2).

As many forms of glomerular disease respond to steroid treatment, ${ }^{17-19}$ we examined whether the ultrastructural changes in the glomeruli of Gstk1 $1^{-1}$ mice could be prevented by treatment with dexamethasone. Male mice treated with daily i.p. injections of dexamethasone and killed at 6 months of age showed no improvement in the glomerular structural features by EM compared with untreated mice. Strikingly, the WT mice treated with dexamethasone showed mild basement membrane changes and a similar level of foot process effacement to the untreated 6-month-old Gstk1 ${ }^{-/-}$ mice (data not shown). Urinary albumin:creatinine ratios were monitored during the dexamethasone treatment. Urine from both WT and Gstk1 $1^{-1-}$ mice treated with dexamethasone had higher albumin:creatinine ratios compared with untreated mice, however, Gstk1 $1^{-/-}$mice continued to have significantly higher ratios than the WT mice with the average over all time points (10-58 days treatment) being $4.4 \pm 1.9 \mathrm{mg} / \mathrm{mmol}$ in Gstk1 $1^{--}$mice compared with $2.3 \pm 0.6 \mathrm{mg} / \mathrm{mmol}$ in WT mice $(n=10$ or 13 for WT and $G s t k 1^{-1-}$, respectively, $\left.P=0.003\right)$.

\section{Influence of GSTK1-1 Deficiency on Circulating Adiponectin}

A previous study indicated that GSTK1-1 has a key role in the multimerization and secretion of adiponectin, ${ }^{20}$ and another study showed that the phenotype of adiponectin-deficient mice is characterized by podocyte foot process effacement in the glomerulus and albuminuria, ${ }^{21}$ two characteristics that are strikingly similar to the phenotype of $G s t k 1^{-1-}$ mice. As a result of this apparent interconnection between GSTK1-1 and adiponectin, we examined the levels of circulating adiponectin, but found only slightly lower (11.6\%) levels in $G s t k 1^{-1-}$ mice $(4.37 \pm 0.22 \mu \mathrm{g} / \mathrm{ml}$ serum compared with $4.94 \pm 0.08 \mu \mathrm{g} / \mathrm{ml}$ in WT mice, $n=5$ or 6 for WT and Gstk1 ${ }^{-1-}$, respectively, $\left.P=0.047\right)$. This brings into question the role in vivo of GSTK1-1 in adiponectin processing and suggests that other unknown proteins are also able to fulfill this function.

\section{DISCUSSION}

We describe here the characterization of mice deficient in GST kappa and the occurrence of microalbuminuria and EM changes in the kidney of these mice. The observation of an increased death rate of male Gstk1 ${ }^{-1-}$ mice associated with urinary tract blockages and inflammation (Figure 6) focused our attention on EM studies of the kidney and urinary tract. These ultrastructural studies revealed significant foot process effacement that was preceded by basement membrane changes, particularly thickening, in the glomerulus that were not evident by light microscopy (Figure 7).

Immunohistochemistry for collagen IV and laminin suggested there were no significant changes in the composition of the GBM (Figure 8). However, the possibility that the distribution of different laminin or collagen IV chains is altered in kappa-deficient mice cannot be excluded. It is possible that the splitting and duplication might result from the persistence of chains present during embryonic stages and/or from protease effects. Although the significance of the morphological GBM changes is not known, a glomerular defect in these mice was confirmed by the observation of microalbuminuria coinciding with the basement membrane changes (Figure 9), suggesting they are functionally significant. These changes did not relate to the accumulation (with age) of immune deposits in the kidneys.

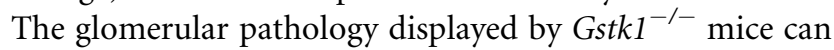
be seen in human glomerulopathies. The basement membrane changes seen in the 3- to 6-month-old Gstk1 ${ }^{-/-}$mice share some features with hereditary nephropathies such as Alport's disease ${ }^{22}$ while the foot process effacement observed at 5-6 months is seen in diseases causing nephrotic syndrome such as minimal change disease, the most common cause of nephrotic syndrome in children. ${ }^{23}$ There is ongoing debate over which component of the glomerulus is responsible for the selective permeability that results in retention of serum proteins during filtration. ${ }^{24}$ Recent studies of genetic causes of nephrotic syndrome in humans and knock-out mice have 
highlighted the contribution of podocyte function and slit diaphragm proteins to this process, ${ }^{19,24,25}$ however, proteinuria can also result from interference with the GBM or with the podocyte-GBM interaction ${ }^{19}$ or from primary GBM defects. ${ }^{26}$ The latter appears to be the case in $G s t k 1^{-1-}$ mice, where the basement membrane changes preceded the foot process effacement in the glomeruli (Table 2) suggesting the basement membrane changes induced podocyte damage. Further, structural changes to podocytes were not necessary for the occurrence of microalbuminuria at 3 months of age, pointing to a contribution from the GBM in permselectivity, as others have reported. ${ }^{26}$

As many patients with idiopathic nephrotic syndrome and albuminuria are treated with corticosteroids, we attempted to reverse the observed changes with corticosteroid treatment of the mice, however, the condition of Gstk1 ${ }^{-/-}$mice was apparently worsened, rather than resolved, by corticosteroids. The extent of foot process effacement observed in Gstk $1^{-/-}$ mice $(40-55 \%)$ is less than that typically observed in minimal change disease ( $\sim 80-90 \%$ ), and patients showing the level of albuminuria displayed in these mice would be unlikely to be treated with corticosteroids and certainly not biopsied for EM examination, thus a direct comparison between humans and $G s t k 1^{-1-}$ mice with respect to steroid responsiveness is inappropriate. Rather it appears that $G s t k 1^{-/-}$mice display subclinical kidney disease, and may be able to offer insights into the early development of glomerulonephropathies.

At the subcellular level GSTK1-1 was originally isolated from the mitochondrial matrix of rat liver, ${ }^{27}$ and has been proposed to have a role in protection against oxidative stress. ${ }^{1,2}$ Our study indicates that GSTK1-1 is a significant contributor to total mitochondrial GST activity in kidney and liver as measured by CDNB activity (Figure 3 ). Decreased mitochondrial function in kidney glomerulus or tubules can contribute to renal dysfunction. This is evident in mice ${ }^{28}$ and humans $^{29}$ with genetic deficiencies of the coenzyme Q biosynthesis pathway. Mitochondrial dysfunction in glomeruli has also been suggested as a crucial factor in the glomerular permselectivity in the acute Finnish-type congenital nephrotic syndrome. $^{30,31}$ The inner mitochondrial membrane proteins Mpv17 and Mpv17L, expressed primarily in proximal tubules rather than glomeruli, ${ }^{32}$ are further examples of mitochondrial proteins associated with renal disease ${ }^{33-35}$ and alterations of the inner ear that resemble Alport syndrome. ${ }^{36}$ In most of these models, the molecules under investigation have been implicated in protecting against oxidative stress, ${ }^{28-32,34}$ a mechanism consistent with the proposed function of GSTK1-1., ${ }^{1,2}$

At the tissue distribution level, the expression of GST kappa within the kidney has been localized by immunohistochemistry primarily to the proximal convoluted tubule, an area of the kidney that is rich in mitochondria, but was not detected in glomeruli, ${ }^{5}$ where we observed the ultrastructural changes. Thus, the presence of glomerular pathology and the lack of changes in the kidney tubules is surprising. A role for GSTK in protection of podocyte mitochondria against oxidative stress would have provided a direct mechanism for the kidney ultrastructural changes observed here in Gstk1 $1^{-/}$mice. It has been suggested that glomerular podocytes may be particularly sensitive to a reduction in respiratory capacity and mitochondrial func$\operatorname{tion}^{28}$ therefore deficiency of GSTK1-1, even if it is only expressed at low levels (ie, below the level of detection by immunohistochemistry), could be sufficient to cause this subclinical phenotype. Interestingly, a recent RNA interference study in C. elegans found that knockdown of GSTK isoenzymes had no effect on reproduction, development, locomotion or life span but a significant reduction in respiration rate was observed. ${ }^{38}$ Decreased mitochondrial function within tubules may also contribute to the observed glomerula nephropathy of Gstk1 $1^{-/-}$mice. Although no structural changes were observed within kidney tubules, a mild deficiency in mitochondrial function, leading to a decrease in ATP production capacity in this mitochondriarich compartment, may impair the proximal tubule's protein resorption capacity, resulting in proteinuria. ${ }^{26,37}$ The change in macromolecule concentrations of the filtrate may result in podocyte damage.

Although a role for GSTK1-1 in protection against oxidative stress in sensitive cell types may be consistent with the kidney phenotype reported here our studies of NQO1 induction and GSH:GSSG content of liver and kidney found no evidence of gross oxidative stress in mice made genetically deficient in GSTK1-1, as was expected from its hypothesized function. This is in contrast to GSTZ1-1-deficient mice, where the accumulation of metabolites from the tyrosine/ phenylalanine catabolism pathway led to constitutive activation of the Keap/Nrf2 pathway, the elevated transcription of Nrf2-responsive genes, and depletion of reduced glutathione in liver. ${ }^{12}$ The mitochondrial location of GSTK11 still leaves open the possibility of an essential role under particular stress conditions, such as adriamycin-induced nephropathy or when other more primary antioxidant defenses such as glutathione peroxidase, glutathione reductase and superoxide dismutase are depleted or overloaded, but this will need to be the subject of further investigations.

The Gstk1 knockout mice developed here have a complete deficiency of GSTK1-1 protein but this does not significantly diminish the total GST activity in the liver or kidney with CDNB, which is dominated by the activity of the alpha, mu and pi class cytosolic GSTs. The other pathologies observed in Gstk $1^{-/-}$mice have not helped to elucidate the function of

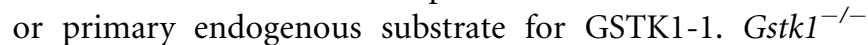
mice were partially protected from thrombus formation that occurred in the heart of male WT mice. Although GSTK1 is expressed at high levels in the heart (Figure 2), ${ }^{2,5}$ no pathological differences were observed within the heart tissue itself. Although a decrease in clotting function in $G s t k 1^{-/}$mice might explain this difference, there were no differences in platelet counts to contribute to this. In contrast, GSTK1-1 is 
not expressed in the spleen of mice or rats (Figure 2), ${ }^{2,5}$ and yet spleen sizes were consistently decreased in $G s t k 1^{-/-}$mice (Table 2), but this was not accompanied by any marked deficiencies in blood cell types as determined by peripheral blood counts. Further studies are required to explain these observations and elucidate the substrate and function of GSTK1-1.

\section{ACKNOWLEDGEMENTS}

We thank Amy Broomfield for performing the immunofluorescence study, Dr Mitali Fadia for pathological assessment of mouse hearts and Professor John Hayes for generously providing antiserum. These studies were under taken with the financial support of NHMRC Grant 418071, an NHMRC RD Wright Fellowship to AB and an NHMRC Australian Biomedical (Peter Doherty) Fellowships to AS and CL.

\section{DISCLOSURE/CONFLICT OF INTEREST}

The authors declare no conflict of interest.

1. Morel F, Rauch C, Petit E, et al. Gene and protein characterization of the human glutathione S-transferase kappa and evidence for a peroxisomal localization. J Biol Chem 2004;279:16246-16253.

2. Jowsey IR, Thomson RE, Orton TC, et al. Biochemical and genetic characterization of a murine class Kappa glutathione S-transferase. Biochem J 2003;373(Part 2):559-569.

3. Robinson A, Huttley GA, Booth HS, et al. Modelling and bioinformatics studies of the human kappa class glutathione transferase predict a novel third glutathione transferase family with homology to prokaryotic 2-hydroxychromene-2-carboxylate (HCCA) isomerases. Biochem J 2004;379(Part 3):541-552.

4. Ladner JE, Parsons JF, Rife $\mathrm{CL}$, et al. Parallel evolutionary pathways for glutathione transferases: structure and mechanism of the mitochondrial class kappa enzyme rGSTK1-1. Biochemistry 2004;43: 352-361.

5. Thomson RE, Bigley AL, Foster JR, et al. Tissue-specific expression and subcellular distribution of murine glutathione S-transferase class kappa. J Histochem Cytochem 2004;52:653-662.

6. Kuhm AE, Knackmuss HJ, Stolz A. Purification and properties of $2^{\prime}$ hydroxybenzalpyruvate aldolase from a bacterium that degrades naphthalenesulfonates. J Biol Chem 1993;268:9484-9489.

7. Thomas KR, Capecchi MR. Site-directed mutagenesis by gene targeting in mouse embryo-derived stem cells. Cell 1987;51:503-512.

8. Lim $\mathrm{CE}$, Matthaei $\mathrm{Kl}$, Blackburn $\mathrm{AC}$, et al. Mice deficient in glutathione transferase zeta/maleylacetoacetate isomerase exhibit a range of pathological changes and elevated expression of alpha, mu, and pi class glutathione transferases. Am J Pathol 2004;165 679-693.

9. Habig WH, Pabst MJ, Jakoby WB. Glutathione S-transferases. The first enzymatic step in mercapturic acid formation. J Biol Chem 1974;249:7130-7139.

10. Mannervik B, MW. Human glutathione transferases: classification, tissue distribution, structure and functional properties. In: PG M, FG N (eds). Advances in Drug Metabolism in Man. The European Commission: Brussels, 1995, pp 407-460.

11. Baker MA, Cerniglia GJ, Zaman A. Microtiter plate assay for the measurement of glutathione and glutathione disulfide in large numbers of biological samples. Anal Biochem 1990;190:360-365.

12. Blackburn AC, Matthaei KI, Lim C, et al. Deficiency of glutathione transferase zeta causes oxidative stress and activation of antioxidant response pathways. Mol Pharmacol 2006;69:650-657.

13. Jo $\mathrm{Yl}$, Cheng $\mathrm{H}$, Wang $\mathrm{S}$, et al. Puromycin induces reversible proteinuric injury in transgenic mice expressing cyclooxygenase- 2 in podocytes. Nephron Exp Nephrol 2007;107:e87-e94.

14. Sharma R, Ahmad H, Singhal SS, et al. Comparative studies on the effect of butylated hydroxyanisole on glutathione and glutathione $\mathrm{S}$ transferases in the tissues of male and female CD-1 mice. Comp Biochem Physiol C 1993;105:31-37.
15. Engle MR, Singh SP, Czernik PJ, et al. Physiological role of mGSTA4-4, a glutathione S-transferase metabolizing 4-hydroxynonenal: generation and analysis of mGsta4 null mouse. Toxicol Appl Pharmacol 2004;194: 296-308.

16. Chen A, Sheu LF, Chou WY, et al. Involvement of immunopathogenic mechanisms in a spontaneously occurring glomerulopathy in mice. Nephron 1998;78:63-72.

17. Bargman JM. Management of minimal lesion glomerulonephritis: evidence-based recommendations. Kidney Int Suppl 1999;70:S3-16.

18. Cattran D. Management of membranous nephropathy: when and what for treatment. J Am Soc Nephrol 2005;16:1188-1194.

19. Asanuma K, Mundel P. The role of podocytes in glomerular pathobiology. Clin Exp Nephrol 2003;7:255-259.

20. Liu M, Zhou L, Xu A, et al. A disulfide-bond A oxidoreductase-like protein (DsbA-L) regulates adiponectin multimerization. Proc Natl Acad Sci U S A 2008;105:18302-18307.

21. Sharma K, Ramachandrarao S, Qiu G, et al. Adiponectin regulates albuminuria and podocyte function in mice. J Clin Invest 2008;118: 1645-1656.

22. Savige J. Hereditary abnormalities of renal basement membranes Pathology 1991;23:350-355.

23. Olson JL. The Nephrotic Syndrome and Minimal Change Disease; Vol. 1, 6th edn, Lippincott Williams and Wilkins: Philadelphia, 2007, pp 136-146.

24. Mathieson PW. The cellular basis of albuminuria. Clin Sci (Lond) 2004;107:533-538.

25. Koop K, Eikmans M, Baelde HJ, et al. Expression of podocyte-associated molecules in acquired human kidney diseases. J Am Soc Nephrol 2003;14:2063-2071.

26. Jarad G, Cunningham J, Shaw AS, et al. Proteinuria precedes podocyte abnormalities inLamb2 ${ }^{-1-}$ mice, implicating the glomerular basement membrane as an albumin barrier. J Clin Invest 2006;116: 2272-2279.

27. Harris JM, Meyer DJ, Coles B, et al. A novel glutathione transferase (1313) isolated from the matrix of rat liver mitochondria having structural similarity to class theta enzymes. Biochem J 1991;278(Part 1):137-141.

28. Peng $M$, Falk $M J$, Haase $V H$, et al. Primary coenzyme $Q$ deficiency in Pdss2 mutant mice causes isolated renal disease. PLoS Genet 2008;4:e1000061.

29. Diomedi-Camassei F, Di Giandomenico S, Santorelli FM, et al. COQ2 nephropathy: a newly described inherited mitochondriopathy with primary renal involvement. J Am Soc Nephrol 2007;18:2773-2780.

30. Holthofer $\mathrm{H}$, Kretzler $\mathrm{M}$, Haltia $\mathrm{A}$, et al. Altered gene expression and functions of mitochondria in human nephrotic syndrome. Faseb J 1999;13:523-532.

31. Solin ML, Pitkanen S, Taanman JW, et al. Mitochondrial dysfunction in congenital nephrotic syndrome. Lab Invest 2000;80:1227-1232.

32. Krick $S$, Shi $S$, Ju $W$, et al. Mpv17 I protects against mitochondrial oxidative stress and apoptosis by activation of $\mathrm{Omi} / \mathrm{HtrA} 2$ protease. Proc Natl Acad Sci USA 2008;105:14106-14111.

33. Weiher H, Noda T, Gray DA, et al. Transgenic mouse model of kidney disease: insertional inactivation of ubiquitously expressed gene leads to nephrotic syndrome. Cell 1990;62:425-434.

34. Binder $\mathrm{CJ}$, Weiher $\mathrm{H}$, Exner $\mathrm{M}$, et al. Glomerular overproduction of oxygen radicals in Mpv17 gene-inactivated mice causes podocyte foot process flattening and proteinuria: $\mathrm{A}$ model of steroid-resistant nephrosis sensitive to radical scavenger therapy. Am J Pathol 1999;154:1067-1075.

35. O'Bryan $\mathrm{T}$, Weiher $\mathrm{H}$, Rennke $\mathrm{HG}$, et al. Course of renal injury in the Mpv17-deficient transgenic mouse. J Am Soc Nephrol 2000; 11:1067-1074.

36. Meyer zum Gottesberge AM, Reuter A, Weiher H. Inner ear defect similar to Alport's syndrome in the glomerulosclerosis mouse model Mpv17. Eur Arch Otorhinolaryngol 1996;253:470-474.

37. Smithies $\mathrm{O}$. Why the kidney glomerulus does not clog: a gel permeation/diffusion hypothesis of renal function. Proc Natl Acad Sci USA 2003;100:4108-4113.

38. Petit E, Michelet X, Rauch C, et al. Glutathione transferases kappa 1 and kappa 2 localize in peroxisomes and mitochondria, respectively, and are involved in lipid metabolism and respiration in Caenorhabditis elegans. Febs J 2009:276:5030-5040. 